ARTICLE

Received 21 Jul 2014 | Accepted 18 Dec 2014 | Published 20 Jan 2015

DOI: $10.1057 /$ palcomms.2014.7

\title{
A study on the state-of-the-art of e-Infrastructures uptake in Africa
}

Fotios Spyridonis ${ }^{1}$, Simon J E Taylor ${ }^{1}$, Pamela Abbott ${ }^{1}$, Roberto Barbera ${ }^{2}$, Amos Nungu ${ }^{3}$, Lars L Gustafsson ${ }^{4}$, Bjorn Pehrson ${ }^{5}$, Omo Oaiya ${ }^{6}$ and Tiwonge Banda ${ }^{7}$

\begin{abstract}
Science is a pioneering method that uses integrated collections of Information and Communication Technologies (ICTs), or e-Infrastructures, to enable scientists across the world to collaboratively work on more and more ambitious projects. Have advances and access to ICTs enabled African e-Infrastructure development? This paper aims to understand the current state of e-Infrastructure uptake in Africa and present some of these initiatives across the African continent by exploring the current landscape emerging from a survey of early consumers. These initiatives are discussed in terms of key projects and user communities that they are addressing. In line with wider perceptions that e-Infrastructures will re-shape the ways research is performed, this study yielded 34 current or planned e-Infrastructure projects across 13 African countries addressing a range of research domains, and identified 7 user communities across these disciplines. Our findings suggest that the African research community is increasingly interested and involved in e-Infrastructure development activities in response to the issue of limited access to dedicated global research and education resources. The study can contribute to the ongoing discussion on how e-Infrastructures can positively promote the research, technological development and innovation potential of developing countries.
\end{abstract}

\footnotetext{
${ }^{1}$ Brunel University London, Department of Computer Science, Kingston Lane, Uxbridge, London, UB8 3PH, UK ${ }^{2}$ Department of Physics and Astronomy, University of Catania, Viale A. Doria, 6, 95125, Catania, Italy ${ }^{3}$ Dar Es Salaam Institute of Technology, United Republic of Tanzania ${ }^{4}$ Karolinska Institutet, Department of Laboratory Medicine, Stockholm, Sweden ${ }^{5}$ School of Information and Communication Technology, KTH Royal Institute of Technology, Brinellvägen 8, Stockholm, Sweden ${ }^{6}$ West and Central African Research and Education Network, 11 Aviation Road Extension P O Box 5744 , Accra, Ghana

7 UbuntuNet Alliance, Off Paul Kagame Road, HB House, Block C, P.O. Box 2550, Lilongwe, Malawi. Correspondence: (e-mail: fotios.spyridonis@brunel.ac.uk)
} 


\section{Introduction}

$t$ the advent of the twenty-first century, the concept of $e$-Science emerged as a pioneering scientific method used around the world to assist in the way scientific research is carried out. The adoption and use of innovative digital platforms consisting of integrated collections of Information and Communication Technologies (ICTs), or e-Infrastructures, lies at the core of e-Science activities throughout the process from the conception of an idea to the production of the final result (Foster, 2002). These have enabled scientists across the world to work together to address more and more ambitious projects. CERN's Large Hadron Collider (LHC) is an example of a truly worldwide research initiative that supports many research projects including the hunt for the Higgs boson particle. This is supported by the Worldwide LHC Grid, possibly the world's largest e-Infrastructure, that supports scientific research based on the massive amounts of data generated by the LHC (http://wlcg.web.cern.ch/). There are many other examples of where e-Infrastructures have assisted smaller international research collaborations to reach more ambitious goals (see later in this paper). However, the challenges of resourcing ICT in Africa are well-known. Although efforts for market liberalization and private investments have initiated major developments in Africa, such as the remarkable increase in mobile phone coverage that has grown from nearly 87 million in 2005 to an estimated 629 million subscribers today, access to the Internet and broadband services remains low in the continent (estimated 3 million users in 2014) (ITU, 2014a).

e-Infrastructures can contribute to the advancement of scientific know-how and can enable ways that this could be exploited. For example, users from different parts of the world, including rural and remote areas, can utilize e-Infrastructures and have access to high-quality research resources, such as specialized instruments in bioinformatics or meteorological data for environmental sciences (Jacq et al., 2007). The availability of bandwidth has hampered African scientific communities from reaching similar benefits, but this is now rapidly changing as the UbuntuNet network's (AfricaConnect, 2014) interconnections unroll across the African continent. It is estimated that almost $20 \%$ of the population will be online by the end of 2014 (up from 10\% in 2010), while Mobile-broadband penetration in Africa will reach close to $20 \%$ in the same year (up from $2 \%$ in 2010) (Sanou, 2014).

What are the implications of these developments for e-Infrastructure uptake in Africa? To try to understand the current state of use of e-Infrastructures, this paper aims to identify and create a picture of various major projects and initiatives in Africa today. This was achieved by reaching out to a wide range of stakeholders in order to understand the current e-Infrastructure scene and the interest in future e-Infrastructure development. The findings of this study will act as input into future e-Infrastructure project support and recommendations and are by no means representative of entire Africa.

Accordingly, this paper is structured as follows. We first introduce the concept of e-Infrastructures and their role in research in the section "e-Infrastructures and their application in e-Science". Related work in the developing world is then presented in the section "Related work in the developing world", while the study methodology is discussed in the section "Study methodology". The results and analysis of the study are then provided in the section "Study results". We conclude with our main findings and their implications in the section "Discussion" and our final remarks in the section "Concluding remarks".

\section{e-Infrastructures and their application in e-Science}

e-Infrastructures are essential drivers of scientific innovation and there is a strong potential to advance development in Africa in this domain. The e-Infrastructure Reflection Group (e-IRG, 2014) defines an e-Infrastructure as “... this new research environment in which all researchers-whether working in the context of their home institutions or in national or multinational scientific initiatives - have shared access to unique or distributed scientific facilities (including high-performance computing (HPC), huge data sets, instrumentation and groupware), regardless of their type and location in the world". Within the scope of this definition, the term e-Infrastructure refers to facilities, resources, systems and related services that are used by ever-increasing communities of scientists and researchers, called Virtual Organizations or Virtual Research Communities (VRCs) to conduct top-level collaborative research in their respective fields (Foster et al., 2001).

The two essential elements of e-Infrastructures are highperformance communication networks and high-performance distributed computing. High-performance communication networks transfer data at high speeds across the world (for example Internet 2 in the United States and GÉANT in Europe). Distributed computing services (such as TeraGrid in the United States and European Grid Infrastructure (EGI) in Europe) share processing power (ranging from supercomputers to desktop PCs), sensors and data storage capacity over these networks and the Internet across VRCs.

VRCs typically use techniques that produce huge amounts of data and knowledge than ever before. Analysing these data requires know-how and resources that can be used by members of various VRCs who are often located in different parts of the world. Access to these can be facilitated through an easy-to-use e-Infrastructure environment, such as high-level user interfaces, for example, a Science Gateway (Teragrid/XSEDE, 2011), that connect to the Grid. A Science Gateway is defined as "a community-specific set of tools, applications, and data collections that are integrated together via a web portal or a desktop application, providing access to resources and services from an e-Infrastructure" (EGI, 2010: 11). In other words, e-Infrastructures can bring together services and resources, while Science Gateways can bring together VRCs and are critical drivers of scientific excellence.

To illustrate how e-Infrastructure services and resources could be accessed through a high-level user interface, the eI4Africa project (eI4Africa, 2012) has developed a Pharmacology Science Gateway (PSG) on behalf and in collaboration with the African Institute of Biomedical Science and Technology. The PSG, which could be found at http://sgw.africa-grid.org/pharmacologyscience-gateway, is an e-Science platform that provides access to resources such as a number of open-source software, HPC facilities, and biomedical data repositories focusing on biomedical and pharmacological sciences. With this Science Gateway, continued contact, mentorship and access to necessary tools and services for laboratories in Africa can be made possible.

e-Infrastructures can therefore help bring together the scientific community to collaborate in their key role towards the design, execution, analysis and storage of science-related activities demanding big data sets. In other words, advances in highperformance networking and HPC infrastructures can help global communities of scientists to collaboratively address more and more ambitious problems than ever before. In order to better exploit the opportunities these technologies can offer in the ways scientific research is carried out, it is essential that these technological developments are adapted to local research needs, such as those required in Africa and in other parts of the world.

\section{Related work in the developing world}

Over the last 10 years, a number of important e-Infrastructures running different types of middleware (that is, novel software that 
sits between the operating systems of computers and allows users to access computers distributed across the network) (CERN, 2014) have been deployed and are currently operational around the developing and emerging world. Given the low diffusion of networking infrastructure across developing countries, e-Infrastructure advances and the efforts to join these to the developed world may be surprising given their otherwise low levels of teledensity (that is, "the number of telephone main lines per 100 inhabitants in a particular country or territory") (ITU, 2014b). Several attempts were made to outline the status of e-Infrastructure projects in the developing world, including the Baltic States (Tautvaisiene et al., 2009), India (Masoni and Cozzini, 2012), or provide a glimpse of a global picture (Andronico et al., 2011). We illustrate these advances in the developing world with two cases from Latin America.

The Latin American e-Infrastructures scene. Initiated in 2003, the America Latina Interconectada Con Europa (ALICE, 2003) project was set up to develop a research network infrastructure within the Latin American region and to link up with European network infrastructures. The project led to the creation of RedCLARA (CLARA, 2003), the first regional Latin American Research and Educational Network, interconnecting 12 National Research and Educational Networks (NRENs) across Latin America and offering connectivity to Europe and other world regions. Both projects have entered their second phase. Starting in 2006 with support from the European Commission, the E-Infrastructure shared between Europe and Latin America (EELA) project aimed to link e-Infrastructure initiatives across NRENs in the two regions by creating a collaborative network and interoperable Grid infrastructure (EELA-2, 2014). EELA has made it possible for Latin American scientists to participate in global science and to adapt existing ICT to further support their scientific needs.

Areas such as e-Health and high-energy physics have already benefited through the EELA project. In the e-Health domain, EELA is supporting several research projects. The GEANT4 Application for Tomographic Emission models nuclear medicine applications and is attempting to speedup up GEANT4 simulations using Grid computing (Jan et al., 2004). User testing of this to study radiotherapy treatments is ongoing in Cuba. As the bandwidth linking Cuba to the rest of EELA is small, the simulations are physically transported to other EELA sites with higher bandwidth, so that the simulations can be submitted to the appropriate VRC for processing. This is an excellent and pragmatic solution to the problem of low bandwidth to make e-Health research infrastructures available to researchers in Cuba.

Along the same lines, the Wide In-Silico Docking of Malaria (WISDOM) project aimed to develop high throughput virtual screening docking-based in-silico drug discovery platforms for neglected and emergent diseases supported by Grid computing (Jacq et al., 2007; Breton et al., 2008). EELA has enabled Latin American countries to take part in global scientific data challenges and to propose related projects, both to the benefit of Latin American scientists. It is a global collaboration and brings together European, South East Asian, Latin American, Mediterranean and South African scientists with internationally available Grid resources.

In the high-energy physics domain, EELA has similarly been supporting a number of projects. With the high-energy physics community growing larger in Latin America, EELA have already selected ALICE and LHCb to run on the infrastructure, with others following soon. The former is building a dedicated heavyion detector to exploit the unique physics potential of nucleusnucleus interactions at LHC energies. The project implements a distributed computing environment that has been used to carry out the production of Monte Carlo data at over 30 sites in four continents, including the EELA Pilot test-bed in Latin America (EELA-2, 2014).

Similarly, the latter project utilizes a number of applications for simulation (for example, GAUSS), digitalization, reconstruction and analysis (for example, DaVinci) that have been used to fully study differences between matter and antimatter. In this experiment, about 700 scientists from 52 institutions around the world are involved, including Latin America (EELA-2, 2014) (LHCb, 2008). The above are examples of work that reflect the exciting global scientific work that Latin American scientists can engage in.

Towards developing African e-Infrastructures. Africa is behind in these developments, mainly due to the breadth and depth of terrestrial ICT infrastructure and the strong dependence on expensive narrowband satellite links (Very Small Aperture Terminal). As a result, with a few exceptions, African universities and research centres often lack access to dedicated global scientific and technological resources, because they are not connected to the global infrastructure consisting of dedicated high-capacity regional networks. The consequence is that research and higher education requiring such access cannot be conducted, and the African continent is not well represented in the global research community. This is witnessed by a world map of the scientific divide where territory size shows the proportion of all scientific papers written by authors living there, as shown on the Worldmapper (2010).

However, this is rapidly changing and there is now a significant amount of activity in developing e-Infrastructures for Africa, particularly at policy levels and network provision. A considerable number of African strategic actors and the European Commission supported joint projects that promoted ICT and e-Infrastructures in the region (ERINA4AFRICA, 2009; EuroAfrica-ICT, 2012; eI-Africa, 2011), with a particular emphasis on three areas: improving e-Infrastructures for university, colleges and research organizations via NRENs; the creation of African VRCs that are linked into global scientific communities; and the ability to test new technologies locally.

Specifically, North Africa is the focus of the EUMEDCONNECT3 programme, which links several North African countries to Europe; the UbuntuNet Alliance is coordinating efforts in the South and the East, while WACREN is the emerging West and Central African Research and Education Network. These efforts are being mirrored on the ground with universities being or planned to be connected by fibre. The other aspect to e-Infrastructures, HPC or Grid computing is less developed than networking. EUMedGrid and EUMedGridSupport were projects investigating this in North Africa and the Mediterranean. SAGrid (South Africa), EPIKH (international-EU), CHAIN-REDS (international-EU) and HP-UNESCO Brain Gain (HP, UNESO) are all examples of projects in the area of developing Grid computing skills and capacity across Africa (ISGTW, 2010).

Even though current work has not fully assessed the implications of these developments to Africa, there are reasons to believe that their effects can lead to significant improvements to the way scientific research is carried out in the continent. It is claimed that e-Infrastructures are powerful enablers of global collaboration and have revolutionized the pursuit of science in many areas, such as physics, bioinformatics, biology and astronomy. e-Infrastructures are key enablers of sustainable development, and may contribute to the prevention of "brain drain" (Bird et al., 2009). So, is there any interest in e-Infrastructures in Africa? For example, are there any African VRCs that currently use or plan to use e-Infrastructures in the future? This paper focuses on two 


\section{Table 1 | Identified current or planned e-Infrastructure projects in Africa}

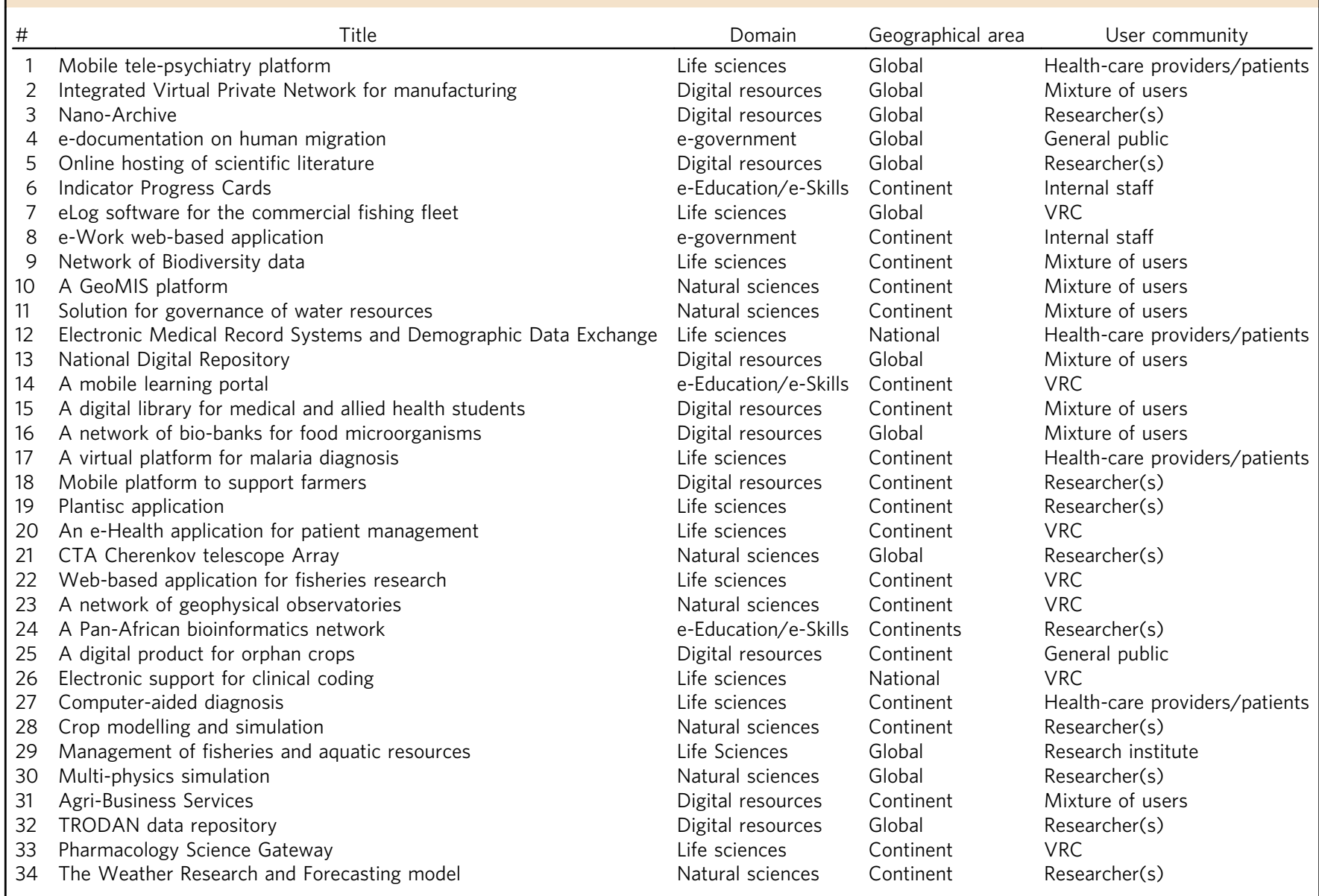

main objectives: (1) study the state-of-the-art of e-Infrastructure projects in Africa, and (2) identify user communities that are early consumers of such projects.

\section{Study methodology}

In addressing the two aforementioned objectives, an online survey was employed that was completed between March 2013 and June 2014. The survey was chosen as the preferred data collection instrument due to the rapid turnaround in data collection and its advantage of identifying attributes of a large population from a small group of individuals (Creswell, 2009).

The survey's design was built on earlier work (EELA-2, 2014) (EGI, 2010) and was developed to elicit information about current or planned e-Infrastructure projects and their users. An introductory page was also prepared briefing a participant about the scope of the study and introducing the concept of e-Infrastructures.

Recruitment of participants. Potential participants were identified using snowballing recruitment through a combination of personal networks of contacts and the European Commission's Community Research and Development Information Service (CORDIS) (CORDIS, 2014). Participants were included in the survey if they had been involved in a project or e-Infrastructurerelated activity, in which at least one African country was a beneficiary.

Survey administration. A wide range of organizations were contacted. These were taken from mailing lists developed over a 6-year period from projects promoting e-Infrastructures in Africa (for example, ERINA4Africa, eIAfrica and PAERIP) and contained more than 10,000 potential participants who have shown interest in wider African ICT policy issues. An invitation to the online questionnaire was first sent in March 2013 followed by two reminders, one at 2 weeks and the other a month later. Participation in the survey was ongoing and remained open for another 14 months, in order to capture future additional participants.

From this, a total of 311 respondents accessed the survey (124 complete; 187 incomplete). Only 124 full responses were used for the analysis, as the remaining were not usable (for example, duplicates and partial answers) and were excluded. The overall response rate was around $39.8 \%$, which is considered typical for online surveys (Evans and Mathur, 2005; Barrios et al., 2011). In terms of the original number contacted this does seem small. However, the mailing lists related to general African ICT policy and not specifically focussed on e-Infrastructures. The majority of the respondents belonged to an educational institution (46.8\%), followed by Small and Medium Enterprises (17.8\%), Non-Government Organizations/Non-Profit Organizations (14.4\%), Governments (10.4\%), research institutions (5.5\%), and other research programmes or projects $(4.8 \%)$. This diversity is reflected in the results presented in the section "Study results".

\section{Study results}

The analysis of the 124 full responses identified 34 usable responses based on which a list of 34 current or planned e-Infrastructure projects in Africa (see Table 1) was generated (each response produced one project). This means that about one 
in four responses was an e-Infrastructure project, which is an important finding with respect to the status of their uptake in Africa. Another important output of the analysis was the identification of seven African user communities involved in e-Infrastructure-related activities. These findings are presented in more detail in the following three subsections.

Geographical distribution of projects. The majority of the projects were reported from Africa with $76.5 \%$. Europe followed with $23.5 \%$ and respondents who were involved in e-Infrastructure-related activities in Africa. From a geographical perspective, the most involved countries represented all African regions: East (5), West (3), Central (2), South (2) and North (1). The United Kingdom, France, Denmark and Belgium represented Northern Europe.

The number of identified projects per country indicated that South Africa (6), Nigeria (5), Tanzania (3) and Uganda (3) were the countries that are most involved in e-Infrastructure developments. This could be explained by the steady growth and increased ICT penetration within these countries that has been taking place over the last 5 years (ITU, 2014a). On the other hand, with the exception of Nigeria, West-Central Africa seemed to be less represented in the e-Infrastructure development scene, while Tunisia was the only North African country that responded with an e-Infrastructure-related project. The case of identifying North Africa's developments in this domain is of particular interest, given the close proximity to Europe and the collaboration with projects such as EUMedGrid and EUMedGridSupport.

Scientific domains of projects. Figure 1 shows the frequency of the scientific domains that the 34 identified projects were broadly

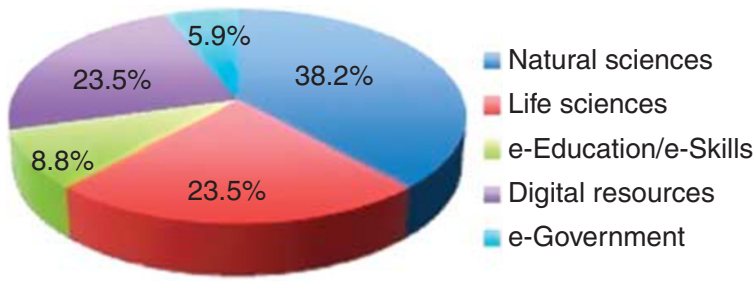

Figure 1 | Distribution of scientific domains of projects (A pie chart showing the distribution of the five identified scientific/research domains that the 34 projects have been broadly grouped into). grouped into. Grouping into these domains was elicited based on the scope and breadth of the project's description provided by a respondent. The most popular domain of scientific activities addressed by the projects was "Natural sciences" followed by "Life sciences". Arguably, this is not a surprising finding as one might expect that most research in Africa would deal with improving the African environment and quality of life.

The "Digital resources" domain was also addressed by a large number of projects. This is an interesting finding, as it demonstrates that projects and resources (for example, Data repositories, e-Libraries) supporting e-Infrastructure development are on the rise in Africa. However, one could argue that they are still at a very early stage given the number of identified projects addressing activities within the "e-Education/e-Skills" domain. Similarly, as expected, "e-government" received less attention among the respondents, an important point for future development given the density of the population living in remote areas.

Breakdown by user communities. Most of the current or planned user communities in Africa identified from the analysis were medium-sized and their activities were either global or spanned several countries. In particular, the respondents reported on smallor medium-sized user communities with a number of members varying from one to more than a million users. Specifically, $11.7 \%$ of the projects addressed large user communities ranging between 50,000 to over a million users, while another $17.7 \%$ targeted no more than 300 users. The former is a particularly interesting finding and shows both the level of interest and the prospects anticipated from the usage of the e-Infrastructure projects.

The analysis revealed seven types of user communities in Africa as the projects' current or planned users. As expected, "Group of researchers" was the most frequent response (26.4\%) across the majority of the projects, followed by VRCs $(20.5 \%)$ and a mixture of the different user communities (21\%). The distribution of findings for the remaining user communities was relatively flat, ranging between $2.9 \%$ and $11.7 \%$ for research institutes or universities, health-care providers or patients, internal staff and the general public.

From a geographical perspective, it is encouraging that most of the projects $(58.8 \%)$ addressed user communities that were spanning more than one country (continent-wide), while $35.2 \%$ were global and only $5.9 \%$ were national. Figure 2 summarizes the above findings about the identified e-Infrastructures user communities in Africa.
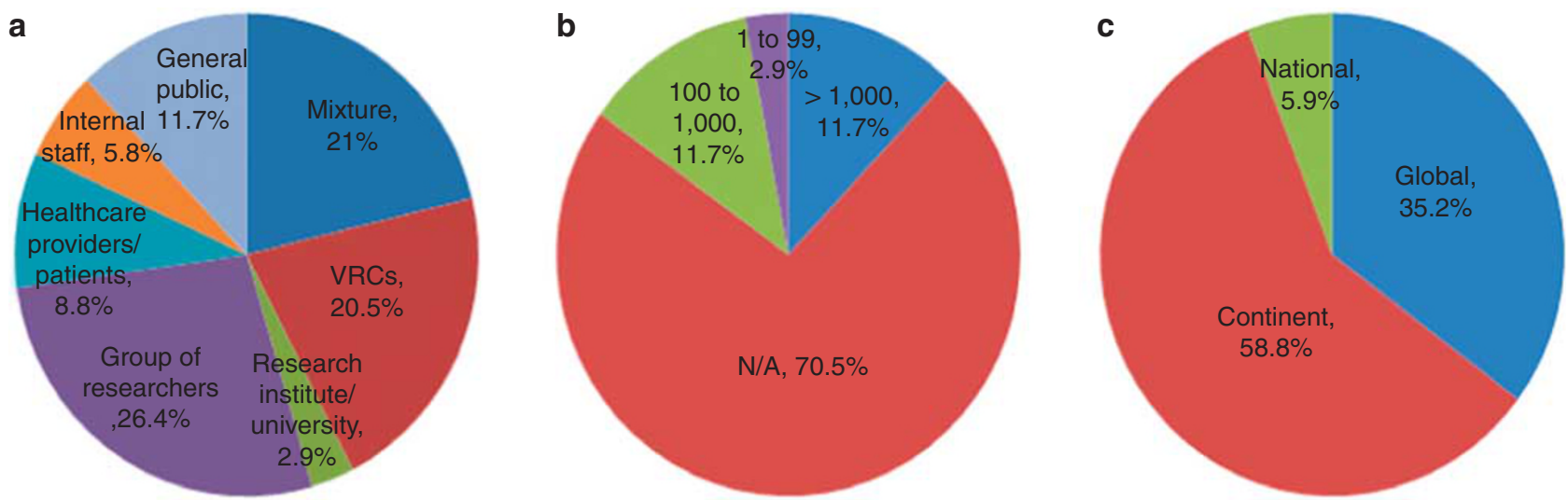

Figure 2 | User communities of e-Infrastructures in Africa. Three different pie charts that show the distribution of (a) the seven identified user communities in terms of current or planned users; (b) the size (in number of users) of the user communities; (c) the current or planned geographic coverage of the user communities. 


\section{Discussion}

Our findings demonstrate that in Africa a respectable $22.4 \%$ of the 58 African countries (as reported by the United Nations (UN, 2012)) have been involved in e-Infrastructure-related initiatives. The examples of South Africa, Nigeria, Tanzania and Uganda show that they are key forces in the emerging interest in developing continent-wide e-Infrastructures. This is particularly encouraging considering the low bandwidth connections limiting such developments in the continent. However, expectations are high, as the rollout of the UbuntuNet network will allow more African countries to participate in worldwide e-Infrastructurerelated projects.

On this basis, can this increasing interest in e-Infrastructures have an impact on future e-Infrastructure development in Africa? Several authors have investigated issues in ICT development in Africa. Four main obstacles to telecommunications diffusion have been identified (Mbarika et al., 2007; Meso et al., 2009b; Mbarika and Byrd, 2009): (1) organizational (poorly informed-IT, governmental monopoly, regulatory frameworks); (2) financial (low GDP, lack of funding for IT infrastructure, lack of financial infrastructure); (3) technological (lack of necessary technical expertise, lack of equipment); and (4) geographical (lack of rural community access).

Accordingly, the results of this work were discussed across several workshops in Africa, involving themes such as raising awareness about e-Infrastructure projects in Africa (Malawi in 2013; 67 participants), demonstrating data infrastructures for e-Science and relevant e-Science projects (Rwanda in 2013; 90 participants), fostering vibrant user communities of practice (Nigeria in 2014; 72 participants) and awareness raising on the use of HPC (Tanzania in 2014; 63 participants). Participants were a mix of policymakers, researchers and potential end-users. Outputs from the workshops indicated that arguably e-Infrastructures are gateways to carrying out international-wide research, collaboration and access to international funding. The following summarizes the major reactions to our results with respect to addressing the above obstacles and further developing e-Infrastructures.

Enabling factors in socio-technological change. In terms of organizational obstacles, the success stories behind e-Infrastructures can have an important effect on national ICT policies of governments, regulatory bodies, institutions within and between countries, and international funding and development agencies. Similarly, given that e-Infrastructure projects tend to address diverse groups of user communities, as this study demonstrated, if these projects were developed in collaboration with appropriate partners, they could act as a vital source of socio-technological knowledge sharing between national and international bodies.

Funding for development and capacity building. Concerning financial obstacles, the benefits of e-Infrastructures can have a major role to play in attracting funding for future development (such as AfricaConnect). Funding for capacity building in Africa is still urgently needed and several international projects are in development to address this. Our survey identified the EU, represented by contributions to projects from the United Kingdom, France, Belgium and Denmark, as being a major source of Euro-African collaboration in the domain of e-Infrastructure development. Given that the EU is a major funder of e-Infrastructures, new funding streams for future development may result from future African e-Infrastructure projects.

Source of technology transfer and expertise. Success stories of e-Infrastructures can have a critical impact on technological obstacles by eliciting expertise, facilitating bargaining for equipment procurement and providing insights to future development. Moreover, the networking, computing, data access and collaboration may be a rich source of technology transfer to future development bodies that could be mediated by successful e-Infrastructure projects. Developers of e-Infrastructures have expertise in delivering complex ICT-based tools to end-users. In the context of an international collaboration between developers, it may be possible to bring in this expertise to avoid potential implementation issues. Appreciating the emerging role of mobile technology in Africa is also important, as it has been shown to be quite different to other developing regions such as Latin America (Meso et al., 2009a).

Promote remote user communities needs and advance crosscultural issues. The problems encapsulated by geographical obstacles can be helped in part by e-Infrastructure projects that would raise awareness of rural community needs, and promote the needs of these remote communities on the international scene. A clear positive impact can be made to culture/cross-cultural issues by utilizing local expertise to advise developers in the area of ICT provision.

The above discussion can be further developed by investigating the following strategies that African countries could adopt to overcome telecommunications diffusion with respect to government and non-government stakeholders and their perceptions (Mbarika and Byrd, 2009): local and foreign investment, development of wireless and satellite infrastructure, appropriately supportive government, non-government (independent bodies) and cross-government policies, appropriately supportive government regulation, building local capacity (skills and expertise) and privatization of telecommunications operators (to combat organizational and policy barriers).

\section{Concluding remarks}

This study aimed to examine whether e-Infrastructure projects have a key role to play in current or future African research activities and e-Infrastructure development. Using examples from Latin America, where e-Infrastructures have been successfully used to support science-related research, we attempted to explore whether e-Infrastructures are being used to make similar contributions to research in Africa. We developed our arguments by presenting a state-of-the-art review of African e-Infrastructure projects that identified 34 current or planned projects spanning five domains of research, and supporting seven different types of user communities. The findings were by no means representative of entire Africa. However, they revealed a number of fascinating potential African e-Infrastructure projects that we see as being indicative of future potential.

Clearly, as with other regions in the developing world, e-Infrastructure projects have major contributions to make to African e-Science, especially in areas such as Natural and Life sciences. However, we do not claim that these will be a "silver bullet" to the problems faced by Africa in accessing dedicated global research and education resources. On the contrary, our findings suggest that e-Infrastructures have the potential to make a significant impact on African e-Science development by simplifying communication across borders and access to scientific information and guidelines required for appropriate services (Royall et al., 2004; Baker et al., 2012; Ardizzone et al., 2012). e-Infrastructures can therefore make vital contributions to the regional development of e-Infrastructures in terms of organizational, financial, technical and geographical obstacles, as well as to ICT policy, ICT infrastructure and ICT implementation. 
Finally, in terms of further work, the eI4Africa project has selected the identified 34 e-Infrastructure projects to lead the deployment of e-Infrastructure projects in Africa. Work is now progressing on the systematic development of socio-technological roadmaps to lead their development. This is following a similar path to e-Infrastructure activities in Latin America and it is hoped that these will bring significant similar benefit to e-Science in Africa. Indeed, since the writing of this paper several key e-Infrastructure demonstrators have been developed in the Africa Grid Science Gateway in both life and environmental sciences. See http://www.eI4Africa.eu for more details.

\section{Acknowledgements}

This work was partially supported by the European Commission project "Teaming-up for exploiting e-Infrastructures' potential to boost RTDI in Africa" (eI4Africa) (no. 312582) and by grants from the Swedish Research Council (2011-7381 and 2013-6710).

\section{References}

AfricaConnect (2014) AfricaConnect. [Online] Available at: http://www.africacon nect.eu/MediaCentre/Pages/New_high-speed_internet_for_African_research_ cooperation.aspx, accessed 8 July 2014 .

America Latina Interconectada Con Europa (ALICE) (2003) ALICE. [Online] Available at: http://alicel.archive.dante.net/, accessed 9 July 2014.

Andronico $\mathrm{G}$ et al (2011) e-Infrastructures for e-Science: A global view. Journal of Grid Computing; 9 (2): 155-184.

Ardizzone V et al (2012) Science gateways for semantic-web-based life science applications. Studies in Health Technology and Informatics; 175 (2012): 119-130.

Baker U et al (2012) 'How to know what you need to do': A cross-country comparison of maternal health guidelines in Burkina Faso, Ghana and Tanzania. Implementation Science; 7 (1): article 31.

Barrios M, Villarroya A, Borrego A and Olle C (2011) Response rates and data quality in web and mail surveys administered to $\mathrm{PhD}$ holders. Social Science Computer Review; 29 (2): 208-220.

Bird I, Jones B and Kee K (2009) The organization and management of grid infrastructures. Computer; 42 (1): 36-46.

Breton V, Jacq N, Kasam V and Hofmann-Apitius M (2008) Grid-added value to address malaria. IEEE Transactions on Information Technology in Biomedicine; 12 (2): 173-181.

CERN (2014) The Grid: Software, Middleware, Hardware. [Online] Available at: http://home.web.cern.ch/about/computing/grid-software-middleware-hardware, accessed 4 November 2014.

CLARA (2003) RedClara. [Online] Available at: http://www.redclara.net, accessed 8 July 2014.

CORDIS (2014) Community Research and Development Information Service. [Online] Available at: http://cordis.europa.eu/fp7/home_en.html, accessed 3 July 2014.

Creswell J (2009) Research Design: Qualitative, Quantitative, and Mixed Method Approaches. SAGE: London.

EELA-2 (2014) EELA-2. [Online] Available at: http://www.eu-eela.eu/first-phase. php, accessed 8 July 2014.

EGI (2010) European Grid Infrastructure. [Online] Available at: https://documents. egi.eu/public/RetrieveFile $?$ docid $=1463 \&$ version $=11$ \&filename $=$ Science_Gate way_Primer_v093_nComments.pdf, accessed 1 July 2014.

eI4Africa (2012) Teaming-Up for Exploiting e-Infrastructures' Potential to Boost RTDI in Africa. [Online] Available at: http://www.eI4Africa.eu, accessed 10 July 2014.

eI-Africa (2011) Cooperation on E-Infrastructures between Europe and Africa. [Online] Available at: http://ei-africa.eu, accessed 5 July 2014.

e-Infrastructure Reflection Group (e-IRG) (2014) e-Infrastructure Reflection Group. [Online] Available at: http://www.e-irg.eu/, accessed 8 July 2014.

ERINA4AFRICA (2009) ERINA4AFRICA. [Online] Available at: http://cordis. europa.eu/project/rcn/98141_en.html, accessed 5 July 2014.

EuroAfrica-ICT (2012) Euro-African Cooperation on ICT Research. [Online] Available at: http://euroafrica-ict.org, accessed 5 July 2014.

Evans J and Mathur A (2005) The value of online surveys. Internet Research; 15 (2): $195-219$.
Foster I (2002) The grid: A new infrastructure for 21st century science. Physics Today; 55 (2): 27-42.

Foster I, Kesselman C and Tueckle S (2001) The anatomy of the grid: Enabling scalable virtual organizations. International Journal of High Performance Computing; 15 (3): 200-222.

ISGTW (2010) Opinion-Africa Grid? [Online] Available at: http://www.isgtw.org/ feature/opinion-africa-grid, accessed 6 July 2014.

ITU (2014a) International Telecommunication Union (ITU). [Online] Available at: http://www.itu.int/en/ITU-D/Statistics/Pages/stat/default.aspx?utm_source = twitterfeed\&utm_medium $=$ twitter.co.jp, accessed 9 July 2014.

ITU (2014b) International Telecommunication Union (ITU). [Online] Available at: https://www.itu.int/osg/spu/intset/focus/bands.pdf, accessed 4 November 2014.

Jacq N et al (2007) Virtual screening on large scale grids. Parallel Computing; 33 (4): 289-301

Jan S et al (2004) GATE: A simulation toolkit for PET and SPECT. Physics in Medicine and Biology; 49 (19): 4543-4561.

LHCb (2008) The LCHb Experiment. [Online] Available at: http://hcb-public.web. cern.ch/lhcb-public/, accessed 8 July 2014

Masoni A and Cozzini S (2012) Applications exploiting e-Infrastructures across Europe and India Within the EU-IndiaGrid Project. In: Maad D S ed. Grid Computing-Technology and Applications, Widespread Coverage and New Horizons. InTech: Croatia.

Mbarika V and Byrd T (2009) An exploratory study of strategies to improve Africa's least developed economies' telecommunications infrastructure: The stakeholders speak. IEEE Transactions in Engineering Management; 56 (2): 312-328.

Mbarika V, Meso P and Musa P (2007) Telecommunications Stakeholders Perceptions of Teledensity: A Comparison of Stakeholders in Latin American Region to those in Sub-Saharan Africa Kona-Waikoloa, Hawaii. The 40th Annual Hawaii International Conference on System Sciences (HICSS 2007).

Meso P, Kaba B, Mbarika V and McCline R (2009a) Regional differences in stakeholders' perspectives on strategies to overcome teledensity limitations: An exploratory comparison of selected latin american and Sub-Saharan African countries. Journal of the Association for Information Science and Technology; 60 (1): 184-200.

Meso P, Mbarika V and Sood S (2009b) An overview of potential factors for effective telemedicine transfer to Sub-Saharan Africa. IEEE Transactions on Information Technology in Biomedicine; 13 (5): 734-739.

Royall J, Bennett M, van Schayk I and Alilio M (2004) Tying up lions: multilateral initiative on malaria communications: The first chapter of a malaria research network in Africa. The American Journal of Tropical Medicine and Hygiene; 71 (2): 259-267.

Sanou B (2014) The World in 2014: ICT Facts and Figures. International Telecommunications Union. [Online] Available at: https://www.itu.int/en/ITU-D/ Statistics/Documents/facts/ICTFactsFigures2014-e.pdf, accessed 25 September 2014

Tautvaisiene G, Mikolaitis S and Puzeras E (2009) E-infrastructure in Baltic states and its application in astrophysics. Memorie della Società Astronomica Italiana; 80: 534 .

Teragrid/XSEDE (2011) Extreme Science and Engineering Discovery Environment. [Online] Available at: https://www.xsede.org/gateways-overview, accessed 7 July 2014

UN (2012) United Nations Statistics Division. [Online] Available at: http://unstats. un.org/unsd/methods/m49/m49regin.htm, accessed 10 July 2014.

Worldmapper (2010) Worldmapper. [Online] Available at: http://www.worldmap per.org/display.php?selected $=205$, accessed 7 July 2014

\section{Additional information}

Competing interests: The authors declare no competing financial interests.

Reprints and permission information is available at http://www.palgrave-journals.com/ pal/authors/rights_and_permissions.html

How to cite this article: Spyridonis F et al (2015) A study on the state-of-the-art of e-Infrastructures uptake in Africa. Palgrave Communications 1:14007 doi: 10.1057/ palcomms.2014.7.

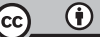

This work is licensed under a Creative Commons Attribution 3.0 International License. The images or other third party material in this article are included in the article's Creative Commons license, unless indicated otherwise in the credit line; if the material is not included under the Creative Commons license, users will need to obtain permission from the license holder to reproduce the material. To view a copy of this license, visit http://creativecommons.org/licenses/by/3.0/ 